\title{
A STUDY FOR THE APPLICATION OF AUTOMATED PLANNING TO MOBILE ASSISTIVE ROBOTS
}

(Short version of the title: AP applied to mobile assistive robots)

\author{
JORGE BREA $^{1}$, CONCEPCIÓN A. MONJE $^{1}$, ANGEL GARCIA-OLAYA $^{2}$ AND CARLOS BALAGUER ${ }^{1}$ \\ ${ }^{1}$ Robotics Lab Group, ${ }^{2}$ Planning and Learning Group \\ University Carlos III of Madrid, \\ Avenida Universidad 30, 28911 Leganés, Madrid, Spain
}

jorge.brea@alumnos.uc3m.es, cmonje@ing.uc3m.es, agolaya@inf.uc3m.es, balaguer@ing.uc3m.es

In the last years Automated Planning (AP) has experimented important advances. In this paper we apply such advances to the field of Mobile Assistive Robots (MAR). In particular, we propose the use of AP to implement the deliberative step between observation and action execution in MAR.

Firstly, we analyse the requirements that allow a MAR to plan navigation and manipulation actions in near realtime. The intention is to build the foundation for a planning module within the SULTAN (Simultaneous User Learning and TAsk executioN) architecture, allowing a MAR to perform Daily Life Activities (DLA) in a human-like environments. Secondly, we apply AP techniques in fully observable, deterministic and static simulated environments with a single MAR. In addition, we analyse and compare the best available satisficing automated planners. The selected planners participate in several experiments to obtain plans for a PDDL domain based on the Tidybot domain. Finally, in order to know how competitive the selected planners are, we compare the experimental results in detail.

Keywords: Automated Planning (AP); Mobile Assistive Robots (MAR); Planning Domain Definition Language (PDDL); International Planning Competition (IPC).

\section{INTRODUCTION}

Robotics is one of the multiple scenarios where greater autonomy is required. Actually, one of the main goals of robotics is to achieve full autonomy. That is why most of the robot control applications involve task exe- 
cution management. Hence, in control architectures for intelligent agents such as autonomous mobile robots, it is particularly desirable the use of a deliberation component: a module for automated planning (AP) or, in brief, a planner (Ghallab, Nau, and Traverso 2004).

The ultimate objective of this planner is to construct plans for execution. This is the so-called control problem in which a model for the problem needs to be specified in terms of: actions, initial situation, goals, and sensors. The outcome of the planner, i.e. the plan, is then delivered to a controller that will perform the actions of the plan: Model $\rightarrow$ Automated Planner $\rightarrow$ Controller.

If a robot has to face a diversity of tasks and/or a variety of environments, then planning will make it simpler to control it, and it will augment the robot's usefulness and robustness. Planning should not be seen as opposed to the reactive capabilities of a robot, whether hand-coded or learned; neither should it be seen as opposed to its learning capabilities. Planning should be closely integrated with these capabilities.

Nowadays, automated planners achieve impressive performance in numerous domains, but a renewed interest in intersecting AP with Robotics has arisen (Bresina et al. 2005), (McGann08 et al. 2008). These papers propose the application of AP technics to efficiently assist a Mobile Assistive Robot (MAR) to build robust plans in deterministic environments.

The rest of the paper is structured as follows. Section II deals with the integration of the AP into a MAR. Section III introduces the building blocks of our proposed planning level. Section IV sketches the characteristics of our experimental environment and Section V shows the experimental results of our approach. Finally, Section VI closes the paper with directions for future research and provides concluding remarks.

\section{INTEGRATING PLANNING INTO A MAR}

Applying AP techniques on the specific domain of robotics has been a topic of research for a long time (Fikes and Nilsson 1971). However, there are few examples of real-world applications. This shortage can be partially blamed on complexity: AP in robotics requires a careful synthesis of navigation and manipulation capabilities.

The specific requirements of planning in robotics, as compared with other application domains of planning, are very demanding: online input from sensors, partial models of the environment and of the robot, noisy and partial knowledge of the state, direct integration of planning with acting, sensing, and learning. These ambitious set of requirements are very complex to handle (Russell 2010). 
Even if the problem to be solved is very simplified, the complexity still remains. This is due to the large branching factor and the overwhelming number of possibilities that can be executed at a time. In fact, what action shall a robot perform first to reach a goal? It is very complex to separate out the relevant actions from those that are meaningless to our purpose.

We define MAR as autonomous mobile robots aimed at assisting people. This definition embraces both the ability to navigate autonomously in indoor environments and the ability to execute manipulation tasks. In this perspective, a MAR shall be able to assist a person in his/her Daily Life Activities (DLA) (Yamazaki 2010).

Since 2000, the Robotics Lab at UC3M is involved in different projects related to the increasing of the quality of life for disabled and elderly people with special needs in their home environment. In this context, the full-sized humanoid robot TEO (Task Environment Operator) is currently being developed to cooperate with humans and other robots in actual collaborative working environments (Martínez et al. 2012). TEO is depicted in Fig. 1.

Complementary to TEO is the Simultaneous User Learning and TAsk executioN (SULTAN) architecture (Balaguer et al. 2011). This is a novel intelligent service robotic system that allows the augmentation of personal capabilities to perform DLA.

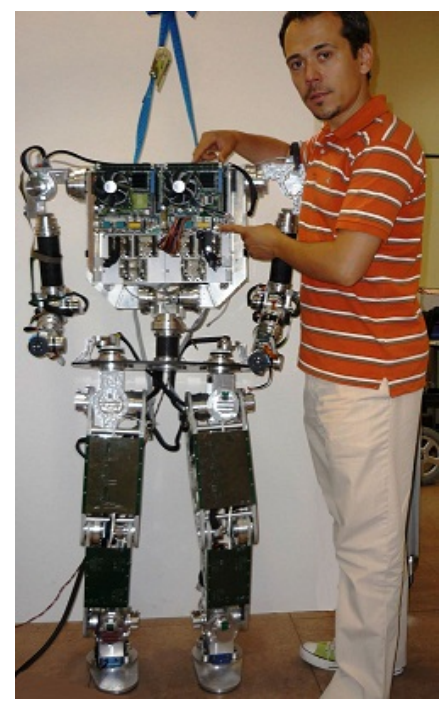

Fig. 1. TEO Concept

SULTAN's goal is to perform intelligent processes (e.g. task planning) to skilfully perform cognitively demanding tasks. For this purpose, we have focused our research on the application of AP techniques in the domain of software developed for MAR, in particular, the design of a planning system. Our goal is to propose 
the use of AP mechanisms to implement part of the deliberative step between observation and action execution in the SULTAN architecture (Fig. 2). Our aim is to contribute to the planning level of the SULTAN architecture by building or re-using a task planner able to solve planning problems in a simulated environment.

This planning module would be in charge of determining the actions that would let the robot achieve its high level goals. For this purpose, we rely on adapting existing general tools, such as domain-independent planners and ready-made planning domains.

\section{PLANNING LEVEL OF THE SULTAN ARCHITECTURE}

One of the weaknesses of most robotic control systems is that they are commonly linked to specific robotic devices. In our approach we solve this problem by separating the robot-platform control from the high level deliberation. In fact, the studied AP techniques could also be applied to low level control but in this research we focus solely on high level task planning.

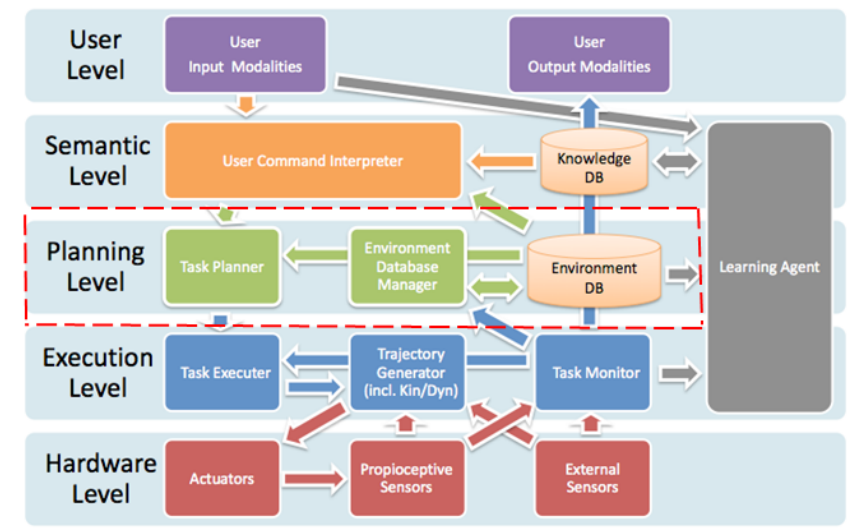

Fig. 2. Planning Level within the SULTAN architecture

By applying AP techniques to the AI of a robot we aim at filling the gap between automated planning theory and practice.

In robotics, task planning should be designed to operate in the real world. It should ideally handle: time, resource allocation, dynamic environments (with uncertainty and partial knowledge) and incremental planning. However, despite existing work on probabilistic planning, most of the planning effort has been made in unreal, deterministic and complete observable environments. The advantage of this approach is purely computational and that is the reason why we have chosen a deterministic environment. 
Moreover, we have decided to address planning tasks in environments without uncertainty, such as those encountered in environments that model simulated worlds. This is basically done for simplification purposes. Otherwise, excessive complexity would prevent the achievement of near real-time task planning. In addition, this is a meaningful and quite used simplification given that the domain has no dead-ends and replanning is possible.

We have already stated that planning time is more important than plan optimality in our experiments. Since MAR actions are uncertain and environments change over time, plans need to be available in quasi realtime. Therefore we are focusing on sub-optimal plans. This way, larger problems will be solved nonoptimally, but more efficiently from an 'execution time' perspective.

The profound knowledge of existing planners' code and internal structure is beyond the scope of this paper. Therefore, the selected planner will be considered as a black box with several inputs (domain and problem) and an output (the target plan).

In summary, the planning level of the SULTAN architecture will integrate all the necessary tools to put AP into practice for a MAR. This toolset comprises:

- A planning domain representation

- A workspace representation

- A valid automated planner

- A set of problems to be solved

The selected tools, acting as building blocks of our planning level, are explained in the following sections.

\section{PLANNING DOMAIN REPRESENTATION}

After the evaluation of other alternatives, we finally focused on domain independent deterministic planning using the standard Planning Domain Definition Language (PDDL) (McDermott et al. 1998). The advantage of this approach is that we can benefit from any PDDL-based planner that had participated in the International Planning Competition (IPC). Moreover, we could benefit from future improvements on these planners very easily. Several planning domains from past IPC editions have been analysed in deep before basing our work on one of them. The intention was to adapt the selected domain to our benefit instead of creating a domain from scratch. 
Several domains modelling robots with manipulation capabilities, like Barman (the robot that prepares cocktails) and Floortile (the robot that paints tiles on the floor), were used at IPC-2011. However, the application of these domains to a MAR is not generic; hence they have been discarded in a first round choice. The second selection rendered three domains that could be applicable to the AI of a MAR. These domains are: Gripper (IPC-1998), Rovers (IPC-2002) and Tidybot (IPC-2011). For the purpose of our experiments, Tidybot covers all our needs. In fact, this domain is very related to the PR2 robot, an R\&D platform developed at Willow Garage and able to plan navigation and complex pick and drop actions.

The Tidybot domain models a household cleaning task, in which one robot must pick up a set of objects and put them into goal locations. Given the amount of actions a MAR could perform according to the Tidybot domain definition, this is the domain selected to carry out our experiments. Table 1 summarizes how the original Tidybot domain has been modified to comply with our requirements and desired goals.

Robots in our domain have a gripper, which moves relative to the robot, up to some maximum radius. Existing objects block the gripper, so that it may be necessary to move one object out of the way to put another one down. In addition, robots can carry one object at a time in the gripper.

\begin{tabular}{|c|c|c|}
\hline $\begin{array}{c}\text { DOMAIN } \\
\text { FEATURES }\end{array}$ & $\begin{array}{c}\text { ORIGINAL TIDYBOT } \\
\text { DOMAIN } \\
\text { (IPC-2011) }\end{array}$ & $\begin{array}{c}\text { TIDYBOT-BASED } \\
\text { DOMAIN } \\
\text { (2013) }\end{array}$ \\
\hline $\begin{array}{c}\text { Amount of actions in the } \\
\text { domain }\end{array}$ & 30 & No \\
\hline $\begin{array}{c}\text { Actions to move the base with } \\
\text { a cart }\end{array}$ & Yes & No \\
\hline $\begin{array}{c}\text { Actions to grasp/ungrasp a } \\
\text { cart }\end{array}$ & Yes & No \\
\hline $\begin{array}{c}\text { Actions to manipulate objects } \\
\text { on the cart }\end{array}$ & Yes & Yes min \\
\hline $\begin{array}{c}\text { Two possible goals for the } \\
\text { same object }\end{array}$ & 30 min & Yes \\
\hline $\begin{array}{c}\text { Time bound when solving } \\
\text { problems }\end{array}$ & Yes & Yes \\
\hline \begin{tabular}{c} 
One arm with one gripper \\
\hline $\begin{array}{c}\text { Able to carry one object at a } \\
\text { time in the gripper }\end{array}$
\end{tabular} & Yes & \\
\hline
\end{tabular}

Table 1. Features of the original Tidybot domain and our experimental domain

Problems from the Tidybot domain are currently a big challenge for many domain-independent planners, as experienced in the Sequential Satisficing track of the IPC-2011. We deem this domain as very complex according to IPC's results and later planning benchmarks presented in ICAPS 2012. The main difficulty lies in 
coordinating the movement of the robot's base and the gripper which makes it a challenging domain for most of the current automated planners.

In our Tidybot-based domain, the robots' behaviour is modelled with nineteen operators that allow a MAR to perform DLA. These operators are summarized in Table 2 (our domain has skipped eleven operators from the original Tidybot domain related to cart usage).

\begin{tabular}{|c|l|}
\hline TYPE OF ACTION & \multicolumn{1}{c|}{ OPERATORS } \\
\hline Base movement actions & $\begin{array}{l}\text { Park, unpark, base-left, base-right, } \\
\text { base-up, base-down }\end{array}$ \\
\hline Gripper movement actions & $\begin{array}{l}\text { gripper-left, gripper-right, } \\
\text { gripper-up, gripper-down }\end{array}$ \\
\hline Object manipulation actions & $\begin{array}{l}\text { get-left, get-right, get-up, } \\
\text { get-down, put-left, put-right, } \\
\text { put-up, put-down, finish-object }\end{array}$ \\
\hline
\end{tabular}

Table 2: Available operators in our Tidybot-based domain

\section{WORKSPACE REPRESENTATION}

With regard to the configuration space, it represents the state of the environment by the Cartesian coordinates of its elements in 2D. The representation for a simple MAR motion and manipulation problem is expressed in workspace coordinates.

The world is structured as a 2D grid, divided into navigable locations and surfaces on which objects may lie. Our planning system considers this geometric environment as composed of: furniture (fixed obstacles), an agent (a MAR with manipulation abilities) and objects (mobile obstacles) that can be manipulated by the agent.

Fixed obstacles take on simple geometric forms: rectangular surfaces were objects may lie. These surfaces may be either tables with four legs (that become an obstacle to the MAR) or tables with a central support (that allow close-in navigation to the MAR). Obstacles raise additional difficulties to plan actions in the environment.

The main advantage of designing such working environment is that it is reusable (with minor changes) for any planar mobile robot. For this reason, the proposed configuration space is robotic-device independent.

Such decomposition has the advantage that it is extremely simple to implement, but it also suffers from limitations. First, it is workable only for low-dimensional configuration spaces, because the number of grid cells increases exponentially with $\mathrm{d}$, the number of dimensions. Therefore, a 3D world has not been simulated due to processing time limitations and the increasing complexity of a new dimension: increasing from N2 in 
$2 \mathrm{D}$ to $\mathrm{N} 3$ in $3 \mathrm{D}$, in an $\mathrm{N} \times \mathrm{N}$ grid. Unfortunately, the difficulty of successfully applying 3D to complex task planning problems still remains.

\section{SELECTING VALID AUTOMATED PLANNERS}

This section explains which one of the many available deterministic automated planners best fits the planning requirements of a MAR.

Environments where DLA are to be performed imply a certain degree of difficulty due to the features of the complex environment and the actions to be executed. If we choose to search for the optimal plan, this complexity would render the search a non-feasible task. This is due to the response time of the currently available automated planners.

In fact, optimal planning would be more useful in practice. But, attending to recent IPC results, optimal planners have not improved too much over the last decade. The constraints they have to face seem a lot harder than those facing satisficing planners.

Therefore, according to the scope and goals of this work, a satisficing deterministic planner is the best option for our purposes. Moreover, since heuristic planning (Bonet and Geffner 1999) is still one of the fastest approaches for AI task planning, we have chosen this search strategy for our work.

For the selection of a planner matching our needs, we have analysed the following set of satisficing heuristic planners:

1. Planners that have been relevant to the research community over the last fifteen years (from IPC-1998 to IPC-2011), i.e., planners winning of with outstanding results at IPC. FF and Metric-FF have not been considered because most of the planners developed after IPC-2000 and IPC-2002 already include the algorithms from FF and Metric-FF inside.

2. Planners that have had the best performance when solving Tidybot domain's problems. Since this domain was submitted at IPC7 (2011), we focus on those satisficing planners competing at this edition of the IPC.

3. Planners developed at the Planning and Learning Group (PLG) of UC3M's Computer Science Department. These planners are SAYPHI (DelaRosa 2006) and CBP (Fuentetaja 2011). SAYPHI has been employed in practice to generate the sequence of high level actions to be executed by autonomous mobile robots and has the advantage of being able to easily incorporate various learning strategies (Quintero et al 2011). CBP (Cost-Based Planner) is a heuristic planner whose objective is obtaining a good balance be- 
tween the quality of the resulting plans and the search time needed to generate them. This objective, together with the good results obtained by CBP in the IPC-2011, turns this planner into a great candidate for the experiments to be carried out in this research.

\section{RANKING THE PRELIMINARY SELECTION OF PLANNERS}

From the broad range of planners mentioned above, we got a reduced list after evaluating the original Tidybot problems as presented at IPC-2011.

For our work, while considering the fast response time and number of solved problems central roles in the evaluation of the competing planners, we will not consider other relatively complex plan metrics involving parallel steps, plan makespan or numerical quantities. In our preliminary selection we have evaluated (a) the response time of each planner and (b) its success rate over 17 original Tidybot problems (only 17 out of 20 available problems were considered because 3 of them were so complex that none of the analysed planners could solve them in 30 minutes in the IPC-2011).

For this preliminary evaluation each planner was given 3 minutes to solve each problem (only mattered whether the problem could be solved or not within 3 minutes). The final 17 planners assessed during our evaluation are (ranked by their results): CBP, LPG, PROBE, SAYPHI, Lamar, BRT, Randward, Fast Downward (FD), SGPlan, LAMA-2011, FD Stone Soup-1, FD Stone Soup-2, FD Autotune-2, FD Autotune-1, LAMA-2008, Roamer and Arvand.

Our experiments show that only three of all the analysed planners fulfil our demanding requirements for a MAR's AI. These promising planners are:

1. CBP: It performed very well overall and therefore became the selected planner for our research.

2. LPG: It achieved a close second position and was used for comparison purposes with regard to CBP's performance.

3. PROBE (Lipovetzky and Geffner 2011): It achieved very good results, but it will be further tested in the future to reach more conclusions. Therefore it is out of the scope of this paper.

Both CBP and LPG are analysed in next section.

\section{VALIDATING THE RESULTING PLANS}

The final building block of our planning level is a tool to make sure planners generate correct plans. This tool is VAL: the automatic PDDL plan validator (Howey 2002). 
This tool is needed to verify that strong constraints and goals of the Tidybot domain are satisfied by any generated plan. This automatic validation tool for PDDL is in fact a sort of constraint checker that guarantees plan quality in terms of its correctness. For our purpose, the basic usage of VAL is appropriate to check whether a plan gets answer YES (correct plan) or NO (incorrect plan).

\section{EXPERIMENTAL TESTBED}

The characteristics of our experimental testbed are explained in this section, together with the selected problems to be solved.

\section{SIMULATION SETUP}

Experiments were conducted on a host machine powered by an AMD Athlon 64 Dual Core 4600 processor, working at 2,4 GHz and equipped with $3 \mathrm{~Gb}$ of RAM. However, not all the resources of the host machine were devoted to the planning tasks. On the contrary, the planning experiments were conducted on a virtual machine installed in the host. The virtual machine simulated a MAR with limited resources, thus imitating a real deployment. This is mainly because of the very limited computational resources on board real robots that prevent them to run the optimal solutions in all but very small domains.

A good example could be a planning module installed in TEO's software architecture. Actually, the planning software would have to share the machine resources with tens of other applications, all of them competing for CPU and memory usage. Actually, in terms of processing speed, TEO is planned to contain two KINO-9454 mini-motherboards (pointed out by one of the authors in Fig. 1). Each of them supporting an Intel Core 2 Duo processor and 4 GB RAM Memory (DDR2) (IEI Technology 2008).

In our testbed, we are assuming that only $1 / 4$ of TEO's processing and memory resources would be devoted to the planning module. That is the reason why our experimental virtual machine was created with the following bounds: a single processor working at 2,4 GHz and 1,5 Gb of RAM memory.

\section{DEMONSTRATIVE SCENARIOS}

Our demonstrative scenario models an office cleaning task, in which one MAR must pick up a set of objects and put them into goal locations. The objective is to re-arrange a set of objects in a room in a pre-specified manner. We ran the tests in a world simulating a typical office environment containing furniture objects. The 
MAR navigates this environment while choosing cell locations to avoid obstacles and manipulates obstacles to free its path.

The MAR in this domain has a gripper, which moves relative to the robot, up to some maximum radius. Existing objects block the gripper, so that it may be necessary to move one object out of the way to put another one down. In addition, the MAR can carry one object at a time in the gripper. The selected planners (CBP and LPG) were allocated a maximum planning time of 3 minutes. And the threshold used to limit every planner's search for solutions was set to one plan (the first plan obtained) for each problem. In most cases, the planners took a time significantly lower than 3 minutes to plan the first solution.

\section{PROBLEMS TO BE SOLVED}

A number of tests have been performed on our Tidybot-based domain to assess the capability of the selected planners. 20 problems have been created by doing a simplification of the original Tidybot problems (as explained before). The tasks in the 20 problems were to collect a number of objects (usually 4) and distribute them on new locations. All problems involved manipulation tasks and path planning. Table 3 details the characteristics of the 20 problems our selected planners had to solve:

\begin{tabular}{|c|c|c|c|c|}
\hline \multirow[b]{2}{*}{$\begin{array}{l}\text { Problem } \\
\text { Number }\end{array}$} & \multirow[b]{2}{*}{$\begin{array}{c}\text { Environment } \\
\text { Size }\end{array}$} & \multirow[b]{2}{*}{ Objects } & \multicolumn{2}{|c|}{ Obstacles } \\
\hline & & & Base & Gripper \\
\hline 1 & $9 \times 9$ & 4 & 15 & 14 \\
\hline 2 & $9 \times 9$ & 4 & 17 & 14 \\
\hline 3 & $9 \times 9$ & 4 & 13 & 14 \\
\hline 4 & $9 \times 9$ & 4 & 13 & 14 \\
\hline 5 & $9 \times 9$ & 4 & 13 & 14 \\
\hline 6 & $9 \times 9$ & 4 & 16 & 14 \\
\hline 7 & $9 \times 9$ & 4 & 13 & 14 \\
\hline 8 & $9 \times 9$ & 4 & 13 & 14 \\
\hline 9 & $9 \times 9$ & 4 & 9 & 14 \\
\hline 10 & $10 \times 10$ & 4 & 9 & 14 \\
\hline 11 & $10 \times 10$ & 4 & 13 & 14 \\
\hline 12 & $10 \times 10$ & 4 & 12 & 14 \\
\hline 13 & $10 \times 10$ & 4 & 16 & 14 \\
\hline 14 & $10 \times 10$ & 4 & 18 & 14 \\
\hline 15 & $10 \times 10$ & 4 & 16 & 14 \\
\hline 16 & $10 \times 10$ & 4 & 13 & 14 \\
\hline 17 & $11 \times 11$ & 4 & 19 & 14 \\
\hline 18 & $11 \times 11$ & 4 & 17 & 14 \\
\hline 19 & $12 \times 12$ & 12 & 35 & 42 \\
\hline 20 & $12 \times 12$ & 8 & 20 & 22 \\
\hline
\end{tabular}

Table 3. Characteristics of the problems to be solved

We set the problem in a robot-task-object domain to solve the challenge of how to plan the necessary actions in different configurations by the same robot. 
Problems with the same size can be quite different in difficulty, so we have generated various problems of the same size for comparison purposes.

\section{PLANNERS' SEARCH MODES}

CBP has been implemented in C, reusing part of the code of METRIC-FF (Hoffmann 2003), and was presented at IPC-2011. CBP can perform heuristic search in the state space using up to 11 different heuristics combined with 20 different search algorithms. However, we just focused our final test on those heuristics that have rendered better results.

CBP works based on the number of actions the planner has needed to solve a given problem. Usually, in cost-based planning the quality of plans is inversely proportional to their cost (Fuentetaja 2011).

Depending on the parameters used to run $\mathrm{CBP}$, the planner can focus either on (a) obtaining the plan as quickly as possible or (b) obtaining a high quality plan in terms of number of actions.

- Configuration to Speed-up the Search: the search algorithm is based on the original METRIC-FF greedy Best First Search (BFS) approach, modified to (a) include look-ahead states and (b) to give priority to helpful actions (Hoffmann 2003) to select the most promising successors in the search.

- Configuration to improve plan quality (configuration by default): the search algorithm adopts the original METRIC-FF Enforced Hill Climbing (EHC) approach, together with the successful $\mathrm{h}_{\mathrm{FF}}$ heuristic (Hoffman 2001) to boost the search. EHC is an incomplete search algorithm (greedy) and thus prone to failure. If EHC fails, CBP falls back into best-first search (a complete A* search).

We have run tests using both configurations and with a time bound of 3 minutes. The search stops at first solution because time response is of the essence for MAR's applications.

With regard to LPG (Gerevini 2002), it is a heuristic planner that was awarded at IPC-2002 (winner) and at IPC-2004 (second place). LPG is a fast planner based on local search techniques and planning graphs, that has evolved over eleven years (1999-2010). It has a variant, LPG-adapt (with versions in 1999 and 2006-07), for fast adaptation and revision of a given plan (also known as replanning in real time).

Both configurations, LPG standard (LPG-td v1.0) and LPG replanning (LPG-Adapt), have been configured to work in speed running mode. 


\begin{tabular}{|c|c|c|c|c|c|c|c|c|c|}
\hline \multirow{2}{*}{$\begin{array}{l}\text { Problem } \\
\text { Number }\end{array}$} & \multirow{2}{*}{$\begin{array}{c}\text { Environment } \\
\text { Size }\end{array}$} & \multicolumn{2}{|c|}{ CBP (speed mode) } & \multicolumn{2}{|c|}{ CBP (quality mode) } & \multicolumn{2}{|c|}{ LPG-td } & \multicolumn{2}{|c|}{ LPG-Adapt } \\
\hline & & $\mathbf{Q}$ & $\mathbf{T}$ & $\mathbf{Q}$ & $\mathbf{T}$ & $\mathbf{Q}$ & $\mathbf{T}$ & $\mathbf{Q}$ & $\mathbf{T}$ \\
\hline 1 & $9 \times 9$ & \multicolumn{2}{|c|}{ Time Out } & 109 & 21 & 187 & 2 & 91 & 3 \\
\hline 2 & $9 \times 9$ & 168 & 49 & 70 & 22 & 66 & 44 & \multicolumn{2}{|c|}{ Time Out } \\
\hline 3 & $9 \times 9$ & 99 & 1 & 38 & 1 & 97 & 8 & 232 & 85 \\
\hline 4 & $9 \times 9$ & 38 & 1 & 24 & 1 & 44 & 65 & 24 & 41 \\
\hline 5 & $9 \times 9$ & 111 & 20 & 59 & 2 & 107 & 12 & 53 & 3 \\
\hline 6 & $9 \times 9$ & \multicolumn{2}{|c|}{ Time Out } & 101 & 9 & 100 & 4 & 86 & 7 \\
\hline 7 & $9 \times 9$ & 125 & 2 & 102 & 92 & 55 & 1 & 43 & 169 \\
\hline 8 & $9 \times 9$ & 51 & 1 & 32 & 1 & 40 & 13 & 79 & 7 \\
\hline 9 & $9 \times 9$ & 76 & 2 & \multicolumn{2}{|c|}{ Time Out } & 61 & 9 & 71 & 12 \\
\hline 10 & $10 \times 10$ & 163 & 3 & 60 & 3 & 68 & 72 & 208 & 13 \\
\hline 11 & $10 \times 10$ & 88 & 4 & 94 & 18 & 127 & 164 & 78 & 24 \\
\hline 12 & $10 \times 10$ & 74 & 1 & 48 & 2 & 337 & 63 & 101 & 89 \\
\hline 13 & $10 \times 10$ & 165 & 39 & 98 & 53 & 170 & 21 & 342 & 32 \\
\hline 14 & $10 \times 10$ & 68 & 1 & 52 & 2 & 62 & 8 & 66 & 20 \\
\hline 15 & $10 \times 10$ & 59 & 1 & 51 & 1 & 45 & 13 & 65 & 13 \\
\hline 16 & $10 \times 10$ & 151 & 131 & 112 & 38 & 121 & 126 & 92 & 2 \\
\hline 17 & $11 \times 11$ & 102 & 16 & 42 & 4 & 182 & 41 & \multicolumn{2}{|c|}{ Time Out } \\
\hline 18 & $11 \times 11$ & 102 & 7 & \multicolumn{2}{|c|}{ Time Out } & 132 & 27 & 78 & 2 \\
\hline 19 & $12 \times 12$ & \multicolumn{2}{|c|}{ Time Out } & \multicolumn{2}{|c|}{ Time Out } & \multicolumn{2}{|c|}{ Time Out } & \multicolumn{2}{|c|}{ Time Out } \\
\hline 20 & $12 \times 12$ & \multicolumn{2}{|c|}{ Time Out } & \multicolumn{2}{|c|}{ Time Out } & \multicolumn{2}{|c|}{ Time Out } & \multicolumn{2}{|c|}{ Time Out } \\
\hline
\end{tabular}

Table 4. Results of CBP and LPG when solving a set of 20 problems: T is average time in seconds (planning times have been rounded to the second). $A$ Time Out value shows problem instances that could not be solved within an adopted time-bound of 3 minutes. Q is average length of the first plan obtained by the planner.

\section{EXPERIMENTAL RESULTS}

We have tested the performance of CBP and LPG in a deterministic environment defined by our Tidybot-based domain. And, for the sake of comparison, we have used the same 20 problems for both CBP and LPG.

We have measured the performance of our testbed according to the average values of three dimensions:

- Time: The time the planner has spent solving the given problem.

- Quality: The number of actions the planner has needed to solve the given problem.

- Reliability: The quality of the satisficing plan in terms of its integrity and approximation to the optimal plan.

Table 4 presents the results of CBP and LPG when solving the proposed 20 problems. Since LPG-td (speed) is stochastic, we ran the planner three times, plotting the median, as its authors usually do. On the contrary, since CBP is deterministic, we run the planner just once (it always obtains the same solution for a given problem). 


\section{PRELIMINARY ANALYSIS}

A preliminary analysis of Table 4 highlights that problems 19 and 20 are a big challenge for the planners. None of the planners' configurations could solve these problems (not even when the planning time was increased to 30 minutes in a later trial). The reason shall be the size of the environment $(12 \times 12)$ and the high amount of obstacles in these problems (see Table 3 to recall each problem's characteristics). These constraints turn these problems into very complex planning tasks because of the limited manoeuvring space of the MAR.

\section{METRICS-BASED ANALYSIS}

In our analysis, we have chosen two metrics to evaluate the planner's performance. For each metric, the planners have been assigned a score for each problem, as explained below. These metrics are:

(1) Planning Time. The time to obtain the first valid plan is scored as follows. The planning time metric's results are presented in Table 5. The best planner in terms of time was judged by summing the scores across all problems.

Results in Table 5 show that any configuration of CBP is able to compute much faster than any configuration of LPG in our testbed. The computational cost of the planning process is lower with CBP and, thus, the running time for solving several different problems is smaller. Moreover, we can state that the CBP planner with speed configuration can achieve the planning task significantly faster on average than the same planner with quality configuration. This conclusion is in line with what was expected from CBP's user guide.

\begin{tabular}{|c|c|c|c|}
\hline \multicolumn{3}{|c|}{ PLANNING TIME - METRIC SCORE (max = 20) } \\
\hline \multirow{2}{*}{ CBP (speed mode) } & CBP (quality mode) & LPG-td & LPG-Adapt \\
& & & \\
\hline 11,1385 & 10,3137 & 5,5777 & 5,4484 \\
\hline
\end{tabular}

Table 5: Scores of the planning time metric. Each planner has got a score 0.0-1.0 for each solved problem. The higher the score, the faster the planner has achieved the plan (max. score =20). Planners score 0 for unsolved problems, otherwise, they score $T^{*} / T$ for a problem, where T is the time for a planner to solve a problem, and $T^{*}$ is the minimum time for any planner to solve the same problem. The overall score of a planner is the sum over 20 problems.

Anyhow, these results are quite satisfactory since the planning time-bound was established at 3 minutes. Each planner could not solve only one problem because of the 3 minutes time constraint. The rest of the unsolved problems (mainly problems 19 and 20) could not even be solved with 30 minutes of planning allowance. 
(2) Planning Quality. The amount of actions of the obtained plan is scored analogously. The planning quality metric's results are presented in Table 6 . The best planner in terms of quality was judged by summing the scores across all problems.

\begin{tabular}{|c|c|c|c|}
\hline \multicolumn{3}{|c|}{ PLANNING QUALITY - METRIC SCORE (max = 20) } \\
\hline \multirow{2}{*}{ CBP (speed mode) } & CBP (quality mode) & LPG-td & LPG-Adapt \\
\hline 9,4701 & 14,4828 & 12,3153 & 11,9585 \\
\hline
\end{tabular}

Table 6. Scores of the planning quality metric. Each planner has got a score 0.0-1.0 for each solved problem. The higher the score the better the plan (it is closer to the optimal plan) (max. score $=20$ ). Planners score 0 for unsolved problems, otherwise, they score $N * / N$ for a problem. Where $N$ is the plan length to solve a problem, and $N^{*}$ is the minimum length over all the planners. The overall score of a planner is the sum over 20 problems.

Here quality is judged via sequential plan length. Since none of the planners can guarantee the optimal plan, the plan length is compared to the best solution found by the planners in the tests. Not surprisingly, the quality of the obtained plans is not always close to the best available solution.

Overall scores show that CBP has obtained significantly good plans when working in quality mode. In fact, the best plans were achieved by CBP (quality) in 8 out of 20 cases.

LPG-Adapt has also solved very well 8 out of 20 problems. However, the rest of its plans were not as good as those achieved by CBP (quality). In any case, we admit there is a limit in the quality of the plans that can be generated with a 3 minutes time-bound.

The first solutions of CBP (quality) are usually better than the ones of LPG and, in general, CBP (quality) finds equal or better quality solutions in less time than LPG.

With regard to CBP running on speed mode, it has not generated any best plan for the given problem set. Again, this conclusion is in line with what was expected from CBP's running mode. Actually, LPG (in any configuration) has achieved significantly better plans on average than those achieved by CBP running on speed mode.

\section{PLAN INTEGRITY ANALYSIS}

Furthermore, we have analysed the quality of the resulting satisficing plans in terms of its integrity and approximation to the optimal plan (the best plan for each problem). 
When it comes to integrity, all plans obtained in our tests have been successfully validated with the VAL tool. This tool has guaranteed that the planners generate plans correctly in terms of our domain's constraints.

However, a closer look at the plans has found that unnecessary actions are included in most of the plans. Mainly in those created by CBP when working in speed mode. We have observed that the first actions in some CBP's plans are, in general, harder to compute since the distance to the goals is greater. This can be caused by the processor and memory limitation of our testbed, together with the problems complexity.

For instance, CBP (in speed mode) has created a plan solving problem \#3 that starts with useless actions. These unnecessary actions move the MAR's arm in the four directions, even though all objects are out of reach, before the base starts navigating.

These results show there is a limit in the quality of the plans CBP can generate in speed mode. This limitation could be overcome by including an additional search strategy when there is available time.

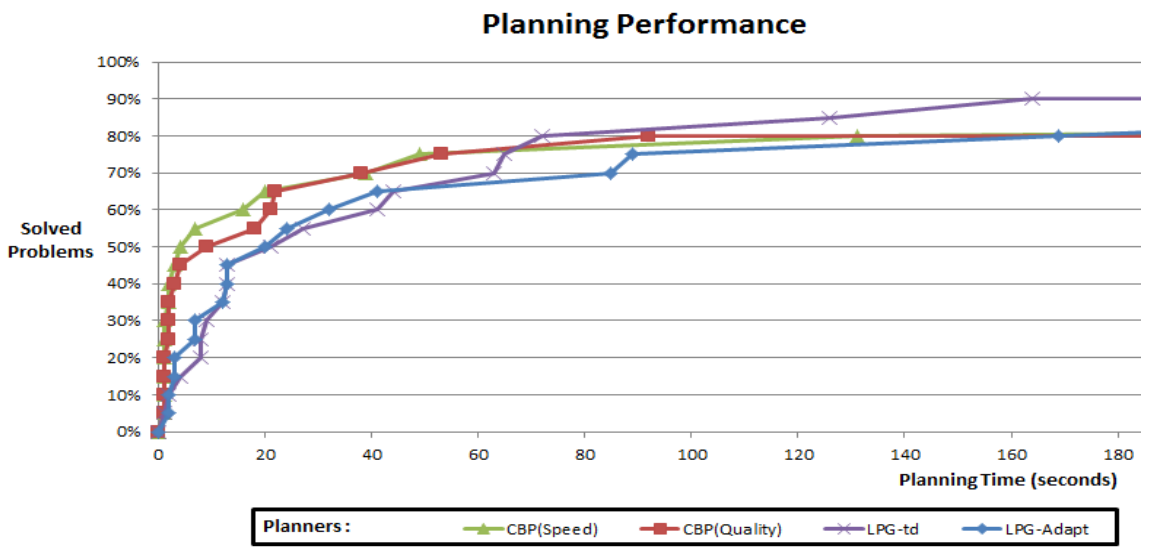

Fig. 3. Planning performance comparison (solved problems vs. planning time)

\section{PERFORMANCE ANALYSIS}

Our final analysis compares the planning performance of the planners. Figure 3 shows the percentage of solved problems in the $y$-axis with regard to the planning time indicated by the $\mathrm{x}$-axis. This helps us finding the probability of a plan to solve a problem in less than a given time, for example: 60 seconds.

Results show that CBP is able to compute a first plan faster than LPG in up to $70 \%$ of the cases. Moreover, CBP scales better than LPG in our Tidybot-based domain. Moreover, we can see that CBP (both in speed and quality modes) can solve 
$75 \%$ of the problems in less than 6 seconds. These means CBP solves almost 10\% more problems than LPG-td (67\%) or LPG-Adapt (65\%) in the same time.

Since none of the planners was able to solve all problems, none of them achieves $100 \%$ in the graph. CBP and LPTAdapt solved all problems except four of them, thus they solved $80 \%$ of the problems. In this case, LPG-td has a higher success rate: it can solve all problems (except three) and thus achieves 90\%. This indicates that LPG-td solves more problems but is not generally the fastest planner to solve a problem.

All in all, given the complexity of the domain and the problems, both CBP and LPG have performed extremely well under the constrained circumstances of a 3 minutes time bound.

\section{CONCLUSIONS AND FUTURE WORKS}

We have presented a comprehensive research for the application of AP techniques to the AI of a MAR. The intention has been finding the building blocks for a high-level planning module that would allow a MAR to perform DLA's in a humanlike environment.

The selected building blocks have been: a planning domain representation (based on the Tidybot domain), a reliable automated planner (CBP) and a set of planning problems in a 2D workspace representation. The Tidybot-based domain presented in this paper is able to exercise the ability of planners to find and exploit planning actions in large and complex problems.

Devising plans for human-like environments implies a certain degree of difficulty. This is due to the features of the complex environments and the actions to be performed. These facts, together with the short response time demanded to MAR, imply the selection of a satisficing deterministic planner for task planning purposes. Only 3 of the 17 analysed planners fulfil the demanding requirements of a MAR's AI. In general, CBP has performed very well in most of the evaluated problems of our Tidybot-based domain. Therefore CBP has become the selected planner for our research. LPG has achieved a close second position and has been used for comparison purposes. PROBE will be evaluated in the future.

Please note that with this evaluation by no means we intend to imply that one particular planner is better than the other in general. There can be other evaluation criteria that give different results and of course results can be very different in other domains, either MAR oriented or not.

In general, we can conclude that the high-level planning module of a MAR (such as the Planning Level within the SULTAN architecture) could greatly benefit from the outcomes of our work and the proposed approach. The path towards the development of a planning level and its integration with other modules (for instance, the Task Executer) has been settled. 
Moreover, we can assure there are excellent tools and planners that are not only free and ready-to-use, but stable and reliable to conduct further experiments. A future step will be the implementation of the task planning on the real robot TEO, from Robotics Lab (UC3M).

\section{REFERENCES}

Balaguer, C., Jardón, A., Monje, C., Bonsignorio, F., Stoelen, M., Martínez, S., et al. 2011. SULTAN: Simultaneous User Learning and Task Execution, and its application in assistive robotics. Workshop on New and Emerging Technologies in Assistive Robotics at IROS. San Francisco (USA).

Bonet, B. and Geffner, H. 1999. Planning as heuristic search: New results. In European Conference on Planning (ECP): 360372.

Bresina, J., Jonsson, A., Morris, P. and Rajan, K. 2005. Mixed-initiative activity planning for Mars Rovers. In International Joint Conferences on Artificial Intelligence: 1709-1710.

DelaRosa, T. B.-O. 2006. Replaying type sequences in Forward Heuristic Planning. In Wheeler Ruml and Frank Hutter, editors, Working notes of the AAAI'06 Workshop on Learning for Search. Boston, MA (USA): AAAI Press.

Fikes, R. E. and Nilsson, N. J. 1971. STRIPS: A new approach to the application of theorem proving to problem solving. In Artificial Intelligence: 189-208.

Fuentetaja, R. 2011. The CBP Planner. In International Conference on Automated Planning and Scheduling (ICAPS) Workshop on International Planning Competition (IPC).

Gerevini, A. S. 2002. LPG: A planner based on local search for planning graphs with action costs. In Artificial Intelligence Planning and Scheduling (AIPS): 13-22.

Ghallab, M., Nau, D. and Traverso, P. 2004. Automated Planning. Theory \& Practice. The Morgan Kaufmann Series in Artificial Intelligence. Elsevier.

Hoffman, J. N. 2001. The FF planning system: Fast planning generation through heuristic search. Journal of Artificial Intelligence Research, 14: 253-302.

Hoffmann, J. 2003. The Metric-FF planning system: Translating “ignoring delete lists" to numeric state variables. Journal of Artificial Intelligence Research (JAIR), 20: 291-341. 
Howey, R. L. 2002. VAL's progress: The automatic validation tool for PDDL2.1 used in the IPC. In International Conference on Automated Planning and Scheduling (ICAPS) Workshop on International Planning Competition (IPC).

IEI Technology Corporation. 2008. KINO-9454 Mini-ITX Motherboard - Used Manual (2.01 ed.).

Lipovetzky, N. and Geffner, H. 2011. Searching for plans with carefully designed probes. In 21th International Conference on Automated Planning and Scheduling.

Martínez, S., Monje, C., et al. 2012. TEO: Full-size humanoid robot design powered by a fuel cell system. An International Journal on Cybernetics and Systems. 43(3): 163-180.

McDermott, D., Ghallab, M., Howe, A., Knoblock, C., Ram, A., Veloso, M., Weld, D., and Wilkins, D. 1998. PDDL--The Planning Domain Definition Language, Technical Report CVC TR-98-003, Yale Center for Computational Vi sion and Control, New Haven (CT, USA).

McGann, C., Py, F., Rajan, K., Ryan, J. and Henthorn, R. 2008. Adaptive control for autonomous underwater vehicles. In 23rd International Conference on Artificial Intelligence, 3: 1319-1324.

Quintero, E., García-Olaya, A., Borrajo, D. and Fernández, F. 2011 . Control of autonomous mobile robots with automated planning. Journal of Physical Agents (JOPHA), 5(1): 3-13.

Russell, S. 2010. Artificial Intelligence: A Modern Approach (3rd ed.). Englewood Cliffs (New Jersey, USA): Prentice-Hall.

Yamazaki, K. U. 2010. System integration of a daily assistive robot and its application to tidying and cleaning rooms. In 2010 IEEE/RSJ International Conference on Intelligent Robots and Systems. 